\title{
Interactive comment on "Quantifying the impact of land cover changes on hydrological extremes in India" by Shaini Naha et al.
}

\section{Anonymous Referee \#1}

Received and published: 2 August 2020

\section{Overview}

The study by Naha et al. aims to assess the role of future land use land cover (LULC) change in altering the nature of hydrological extremes in the Mahanadi river basin in India, under different future scenarios, described by a combination of socio-economic pathways (SSPs) and representative concentration pathways (RCPs). The manuscript deals with an important topic in a region sensitive to hydrological extremes. It is, in general, well-written and easy-to-read. However, several aspects of the study, including novelty, introduction, and methodology (specifically the sensitivity analysis and calibration) are either not well designed or incompletely described. I cannot recommend the publication of this study until these issues are satisfactorily addressed. 
1. Introduction: One of the main issues is the lack of novelty in the presented research, outside its contribution as a case study. The authors need to clearly bring out the novelty of their research compared to the existing research (cited in the study) which have already shown that model parameters have an impact on future land use change studies.

- Line 50 - 55: "However, the exact role of LULC changes in modifying river discharge is still elusive (Rogger et al., 2016) and therefore, remains a challenge to isolate the sole impacts of land use changes on hydrology of a river basin (Tsarouchi and Buytaert, 2018)". These claims are very vague. What are the specific challenges and how does the present study aims to overcome them?

- Line 105 - 110: What is missing in these studies which the authors have solved in the presented study? This is not very clear.

- Line 115 - 120: The research questions are very vague. None of the research questions pertain to the central question question: impact of LULC change on hydrological extremes. Instead it focuses on the modeling part.

2. Design of Study and Methodology: I have serious issues with the design of the study. As it stands, it reads like two different studies which are unrelated to each other: 1) quantify impact of parameter uncertainty and 2) quantify impact of lulc cover change. However, there is no attempt to connect these two objectives. It is well known that parameter uncertainty will have an impact on the output of hydrologic models. However, how does this specifically relate to LULC change studies? This is not clear

- It is not clear as to why only soil parameters are selected for sensitivity analysis. I would imagine that the selection of the hydrologic model should be

Printer-friendly version

Discussion paper
Interactive comment 
based on how sensitive the model parameters are to vegetation parameters which LULC. In fact, this should be the first step to understand the behavior of the model to changes in LULC. This information is missing.

- Model calibration and Pareto ranking is poorly described. What are the specific steps involved in assigning the ranks?

- I am not sure if I understood this correctly but only a range of parameter values are provided for each LULC type. It would be better to provide the parameter values that are used for each LULC type to see if they are physically consistent.

\section{Results, Discussion and Conclusions:}

- Figure 6b: The calibrated parameter ranges are too wide (equifinality).I am not sure why calibration was not able to narrow the range of parameters here. The authors need to discuss why the ranges are not more constrained even after calibration. The discussion in lines 615 - 635.

- Figure 9: The issue of using only related parameters for calibration is reflected in the water balance components. Evaporation, which I assume is dependent on vegetation parameters, shows very little change (less than 8 $\%)$, while soil moisture exhibits changes large variations. This makes it very difficult to discern the impact of LULC changes alone. I recommend that the authors also present results with only the best parameter. Of course, I assume here that actual evaporation is explicitly modeled in VIC and is not an input.

- Line 655 - 670 - Many of the points under major findings are a repetition of the results and do not represent substantial or novel conclusions. 